\title{
Local analytical sensitivity analysis for design of continua with optimized 3D buckling behavior
}

Pedersen, Niels Leergaard; Pedersen, Pauli

Published in:

Structural and Multidisciplinary Optimization

Link to article, DOI:

10.1007/s00158-017-1755-8

Publication date:

2018

Document Version

Peer reviewed version

Link back to DTU Orbit

Citation (APA):

Pedersen, N. L., \& Pedersen, P. (2018). Local analytical sensitivity analysis for design of continua with optimized 3D buckling behavior. Structural and Multidisciplinary Optimization, 57(1), 293-304.

https://doi.org/10.1007/s00158-017-1755-8

\section{General rights}

Copyright and moral rights for the publications made accessible in the public portal are retained by the authors and/or other copyright owners and it is a condition of accessing publications that users recognise and abide by the legal requirements associated with these rights.

- Users may download and print one copy of any publication from the public portal for the purpose of private study or research.

- You may not further distribute the material or use it for any profit-making activity or commercial gain

- You may freely distribute the URL identifying the publication in the public portal 


\title{
Local analytical sensitivity analysis for design of continua with optimized 3D buckling behavior
}

\author{
Niels L. Pedersen · Pauli Pedersen
}

Received: date / Accepted: date

\begin{abstract}
The localized analytical sensitivity for eigenfrequency is extended to the non-linear problem of $3 \mathrm{D}$ continuum buckling analysis. Implemented in a finite element approach the inherent complexity of mode switching and multiple eigenvalues is found not to be a practical problem. The number of necessary redesigns is of the order 10-20 as illustrated by a specific example, where also different cases of stiffness interpolation are exemplified.
\end{abstract}

Keywords Sensitivities · buckling · analytical · optimization · FE

\section{Introduction}

The book by Haftka et al (1990) include references to early papers on optimization that involves buckling as an objective or as a constraint and the subject still needs further research. In the papers $\mathrm{Wu}$ and Arora (1988), Mróz and Haftka (1994), Kleiber and Hien (1997) and in the review Ohsaki (2005) extensive formulations of sensitivity analysis for non-linear problems are presented and discussed, including problems where stability is essential. The shown examples in these references are still concentrated on structural

Niels L. Pedersen

Dept. of Mechanical Engineering, Solid Mechanics

Technical University of Denmark

Nils Koppels Allé, Building 404, DK-2800 Kgs. Lyngby, Denmark

E-mail: nlp@mek.dtu.dk

Pauli. Pedersen

Dept. of Mechanical Engineering, Solid Mechanics

Technical University of Denmark

Nils Koppels Allé, Building 404, DK-2800 Kgs. Lyngby, Denmark models such as trusses and frames. In the discussion of Bruyneel et al (2008) on design of continua it is in the conclusion stated that "buckling optimization is a very difficult problem and the reasons for slow convergence are multiple". In the recent papers by Dunning et al (2016), Luo and Tong (2015), Sørensen et al (2014) and Colson et al (2010) the discussion is continued including aspects of sensitivity analysis, choice of optimization method, and the possibility of several buckling modes. The present paper is intended to be short and add to the discussion of Bruyneel et al (2008). In spite of the fact that buckling of trusses and frames are of major importance, the present research concentrate on continua.

In the present paper focus is on the sensitivity analysis. The optimization method chosen is an optimality criterion method but this is of minor importance as sensitivities are of more general value. Each optimization step is based only on the buckling mode of the lowest eigenvalue and still "convergence" is obtained in 10-20 redesigns, although influence from switching of buckling mode is seen. Including several buckling modes as in the cited references may improve convergence further, but is not attempted in the present paper, which concentrate on the formulation for sensitivity analysis and the discussion of the basis for the involved eigenvalue problem.

The main difference between the cited references and the present formulation of the eigenvalue problem for buckling determination (estimation) relates to the involved matrices. In the references the initial global stiffness matrix is applied, i.e., a secant stiffness matrix based on Green-Lagrange strains to obtain non-linear static equilibrium is not involved. The eigenvalue problem is in the present paper stated as a linear extrapolation from a geometrically non-linear state, with two 
matrices from this state which describe the resulting tangent stiffness matrix.

For a given continuum (design) with given load distribution and specified support conditions, a buckling load factor may be determined (estimated) by solution of an eigenvalue problem as described in Cook et al (2002) based on linear elastic analysis. This formulation involves the initial global stiffness matrix that only depends on the current design. However, in the present formulation the necessary data for the eigenvalue problem is obtained by the solution of a static equilibrium of a geometrical non-linear elastic problem, iteratively applying current secant and tangent stiffness matrices based on Green-Lagrange strains. This geometrical nonlinear approach in reality simplifies the sensitivity analysis for the eigenvalue problem, and include the possibility for changed relations between individual stresses.

The eigenvalue problem includes the tangent stiffness matrix separated in the stress stiffness matrix and the remaining part of the tangent stiffness matrix. For a tetrahedron element analytical expressions for these matrices are available in Pedersen (2006) and are applied for the presented research. It is directly seen that especially the stress stiffness matrix is rather simple. The eigenvalue problem is solved by the method of subspace iteration giving in addition to the eigenvalue (load factor) also the corresponding buckling mode. With this mode a Rayleigh quotient is equal to the eigenvalue and the formulation is presented in energy terms that may be directly accumulated from element energies, remembering that the element stress stiffness matrices are indefinite.

Two aspects of the eigenvalue problem simplify the sensitivity analysis, defined as change in load factor (eigenvalue) as a function of a specific element design parameter. At first the gradient of the Rayleigh quotient is stationary with respect to the eigenmode, .i.e., the change of buckling mode will not influence the first order derivative. Secondly, the accumulation from element energies, with only local explicit influence from design parameter to the corresponding element matrices, implies that local analytical sensitivity analysis can be derived and applied.

An optimality criterion is closely related to the sensitivity gradient and a heuristic numerical approach determine a redesign towards an increased load factor, assume no mode switching. The number of needed redesigns is of the order 10-20 and mainly the computational demanding part is the chosen implicit nonlinear elastic solutions after each redesign, which with Newton-Raphson iterations involves a Gauss elimination for each iteration. The presented example in 3D have close to 100000 design variables, close to 100000 degrees of freedom and for the linear equations a bandwidth close to 1000 , still being computationally acceptable.

The layout of the paper is as follows: Section 2 describe mathematically the essentials of the stress stiffness matrix for a FE tetrahedron and Section 3 describes the formulation of buckling as an eigenvalue problem. Localization of results for sensitivity analysis is shown in Section 4 and are in Section 5 applied to obtain an optimality criterion that a design must satisfy when the size limits for design parameters are not active. A numerical example is finally discussed in Section 6, including different cases of stiffness interpolation. The obtained results should be of interest for research related to topology optimization, although 0-1 solutions are not attempted.

\section{Stress stiffness matrix}

The stress stiffness matrix $\left[S_{\sigma}\right]$ is a part of the tangent stiffness matrix $\left[S_{t}\right]$. The remaining part we index as the gamma stiffness matrix $\left[S_{\gamma}\right]$, that in Section 5.1.2 of Crisfield (1991 and 1997) is indexed with $t 1$. With zero displacement gradients $\left[S_{\gamma}\right]$ simplifies to the stiffness matrix for linear elasticity. The matrix $\left[S_{\gamma}\right]$ is positive definite.

$\left[S_{t}\right]=\left[S_{\gamma}\right]+\left[S_{\sigma}\right]$

All these stiffness matrices are symmetric, and are in Pedersen (2006) available by analytical expressions for the simple four node tetrahedron element. From these expressions it is seen that $\left[S_{\sigma}\right]$ is proportional to a factor on the stresses, i.e., a factor on the load distribution in a tangential extrapolation. Further simplifications are seen with presentation on the directional level:

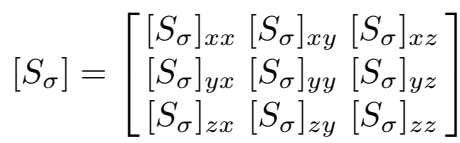

$$
\begin{aligned}
& =\left[\begin{array}{ccc}
{\left[S_{\sigma}\right]_{x x}} & {[0]} & {[0]} \\
{[0]} & {\left[S_{\sigma}\right]_{y y}=\left[S_{\sigma}\right]_{x x}} & {[0]} \\
{[0]} & {[0]} & {\left[S_{\sigma}\right]_{z z}=\left[S_{\sigma}\right]_{x x}}
\end{array}\right]
\end{aligned}
$$

and the $4 \times 4$ matrix $\left[S_{\sigma}\right]_{x x}$ is

$$
\begin{aligned}
{\left[S_{\sigma}\right]_{x x}=} & \sigma_{x x}\left[T_{x x}\right]+\sigma_{y y}\left[T_{y y}\right]+\sigma_{z z}\left[T_{z z}\right]+ \\
& \sigma_{x y}\left(\left[T_{x y}\right]+\left[T_{x y}\right]^{T}\right)+\sigma_{x z}\left(\left[T_{x z}\right]+\left[T_{x z}\right]^{T}\right)+ \\
& \sigma_{y z}\left(\left[T_{y z}\right]+\left[T_{y z}\right]^{T}\right)=\left[S_{\sigma}\right]_{x x}^{T}
\end{aligned}
$$


where the six basis matrices $\left[T_{i j}\right]$ are determined directly from the initial nodal positions. The simple expressions for the stress stiffness matrix by (3) and (2) is directly taken from the total tangent stiffness matrix in Pedersen (2006). The remaining part of the tangent stiffness matrix is $\left[S_{\gamma}\right]$ as defined in (1) by $\left[S_{\gamma}\right]=$ $\left[S_{t}\right]-\left[S_{\sigma}\right]$.

\section{Buckling as an eigenvalue problem}

With the tangent stiffness matrix available for a $\mathrm{FE}$ model, buckling may be determined as described in Cook et al (2002). In buckling two close equilibrium states are possible for the same load.

An initial non-linear obtained reference state is assumed, corresponding to the load $\{A\}_{i}$ and is obtained by a solution of the geometrical non-linear elastic equilibrium $\left[S_{s}\right]_{i}\{D\}_{i}=\{A\}_{i}$, where the system secant stiffness matrix is non-symmetric and depending upon the displacement vector $\{D\}_{i}$. An iteratively obtained solution is given the index $i$ to indicate the dependence on the chosen prescribed load distribution $\{A\}_{i}$, which might include imperfections and may be changed/scaled when closer knowledge to the actual optimization problem is obtained. At convergence for the geometrical non-linear static equilibrium the tangent stiffness matrix is obtained. Then for further load increment (extrapolation), this matrix is assumed fixed, i.e., both $\left[S_{\gamma}\right]$ and $\left[S_{\sigma}\right]$ do not depend on the further displacements. This is the background for the simple sensitivity analysis.

Different solution approaches are possible, also without focus on the secant stiffness matrix. Here the RayleighRitz approach is applied and a system residual $\{R\}$ is defined from

$$
\{R\}=\left[S_{s}\right]\{D\}-\{A\}
$$

and then iteratively update the estimate $\{D\}$ by $\{\Delta D\}$ found from

$$
\{R\}+\{\Delta R\}=\{0\} \Rightarrow\{\Delta R\}=-\{R\}=\left[S_{t}\right]\{\Delta D\}
$$

where the total tangent stiffness matrix $\left[S_{t}\right]$ is symmetric and also depending upon the current displacement vector $\{D\}_{i}$. The update is done on the system level, so that the system tangential stiffness matrix and the system residual vector can be assembled in the usual finite element manner, but without needing to assemble the system secant stiffness matrix that is non-symmetric, i.e., $\{R\}=\sum_{e}\left(\left[S_{s}\right]_{e}\{D\}_{e}-\{A\}_{e}\right)$.
A determined solution $\{D\}_{i}$ gives the gamma stiffness matrix $\left[S_{\gamma}\right]$ and the stress stiffness matrix $\left[S_{\sigma}\right]$ by

$$
\begin{gathered}
{\left[S_{s}\right]_{i}\{D\}_{i}=\{A\}_{i} \Rightarrow\{D\}_{i} \Rightarrow} \\
\gamma_{i} \Rightarrow\left[S_{\gamma}\right]_{i} \text { and } \sigma_{i} \Rightarrow\left[S_{\sigma}\right]_{i}
\end{gathered}
$$

For an extrapolated load step, the tangent stiffness matrix is added the effect of scaled (increased) stress by the factor $\lambda_{i}$ for estimation of the buckling load. With the definition $\lambda=1+\lambda_{i}$ this gives

$$
\begin{aligned}
& \left(\left(\left[S_{\gamma}\right]_{i}+\left[S_{\sigma}\right]_{i}\right)+\lambda_{i}\left[S_{\sigma}\right]_{i}\right)\{\Delta D\}_{i+1}= \\
& \left(\left[S_{\gamma}\right]_{i}+\left(1+\lambda_{i}\right)\left[S_{\sigma}\right]_{i}\right)\{\Delta D\}_{i+1} \Rightarrow \\
& \left(\left[S_{\gamma}\right]_{i}+\lambda\left[S_{\sigma}\right]_{i}\right)\{\Delta D\}_{i+1}=\lambda\{\Delta A\}_{i+1} \\
& \left(\left[S_{\gamma}\right]_{i}+\lambda\left[S_{\sigma}\right]_{i}\right)\left(\{\Delta D\}_{i+1}+\{\Delta\}\right)=\lambda\{\Delta A\}_{i+1}
\end{aligned}
$$

where $\{\Delta\}$ is the buckling mode from $\{\Delta D\}_{i+1}$. The difference of these two equations gives an eigenvalue problem

$\left(\left[S_{\gamma}\right]_{i}+\lambda\left[S_{\sigma}\right]_{i}\right)\{\Delta\}=\{0\} \Rightarrow$ the eigenpair $\lambda_{1},\{\Delta\}_{1}$

The critical load $\{A\}_{C}$ corresponding to the buckling mode $\{\Delta\}_{1}$ is

$\{A\}_{C}=\lambda_{1}\{A\}_{i}$

where $\lambda_{1}$ is the lowest eigenvalue, with $\lambda_{1}>1$ (which is obtained by scaling of $\{A\}_{i}$ ). (If $\lambda_{1}=1$ the tangent stiffness matrix is singular and $\{\Delta\}_{1}$ not defined).

\section{Localization of sensitivity analysis}

The eigenvalue problem (8) presented without index $i$ is

$$
\left(\left[S_{\gamma}\right]+\lambda\left[S_{\sigma}\right]\right)\{\Delta\}=\{0\}
$$

that is pre multiplied by the transposed buckling eigenmode $\{\Delta\}^{T}$ to

$U_{\gamma}+\lambda U_{\sigma}=0$ with the defined energies

$U_{\gamma}:=\{\Delta\}^{T}\left[S_{\gamma}\right]\{\Delta\}$ and

$U_{\sigma}:=\{\Delta\}^{T}\left[S_{\sigma}\right]\{\Delta\}$

and the Rayleigh quotient 
$\lambda=-\frac{U_{\gamma}}{U_{\sigma}}$

The sensitivity of the buckling eigenvalue with respect to the density $\rho_{e}$ of the local element $e$ is

$\frac{\partial \lambda}{\partial \rho_{e}}=-\frac{\partial \frac{\widehat{U_{\gamma}}}{U_{\sigma}}}{\partial \rho_{e}}-\frac{\partial \frac{U_{\gamma}}{U_{\sigma}}}{\partial \Delta} \frac{\partial \Delta}{\partial \rho_{e}}=-\frac{\partial \frac{\widehat{U_{\gamma}}}{U_{\sigma}}}{\partial \rho_{e}}$

simplified by the stationarity of the Rayleigh quotient with respect to change of the eigenmode and applying a hat notation for gradients with unchanged eigenmode, see Pedersen and Pedersen (2015) with reference to Wittrick (1962).

Note for the energies in (11), that the stiffness matrices are based at the solution $\{D\}_{i}$ for the non-linear elastic problem while the buckling mode $\{\Delta\}$ is the eigenmode. The stationarity of $\lambda$ as a function of buckling mode is not an assumption of independence but only related to the first order partial derivative. In the linear extrapolation from the non-linear static equilibrium, the tangent matrices $\left[S_{\gamma}\right]$ and $\left[S_{\sigma}\right]$ are fixed and obtained by the current determined displacement vector $\{D\}_{i}$ from $\left[S_{s}\right]_{i}\{D\}_{i}=\{A\}_{i}$, but $\{D\}_{i}$ is not explicitly involved in the sensitivity analysis. This is the background for the simple results obtained with the energy eigenvalue formulation where the buckling mode $\{\Delta\}$ and not the displacement vector $\{D\}_{i}$ is involved. Every new redesign is initiated with a non-linear static analysis, iteratively determining $\left[S_{s}\right]_{i}$. From several examples it is seen that almost monotonous convergence is obtained with small redesign changes. Influence from multiple buckling modes that have equal or nearby eigenvalues are taken care of in later redesigns without extended sensitivity analysis for non-single eigenmodes. Also mode switching is seen for the cases when applying the method of subspace iterations to obtain more than one eigenvalue.

Further differentiation of the Rayleigh quotient give

$\frac{\partial \lambda}{\partial \rho_{e}}=-\frac{\partial \widehat{U}_{\gamma}}{\partial \rho_{e}} \frac{1}{U_{\sigma}}+\frac{\partial \widehat{U}_{\sigma}}{\partial \rho_{e}} \frac{U_{\gamma}}{U_{\sigma}^{2}}=-\frac{1}{U_{\sigma}}\left(\frac{\partial \widehat{U}_{\gamma}}{\partial \rho_{e}}+\lambda \frac{\partial \widehat{U}_{\sigma}}{\partial \rho_{e}}\right)$

where the energies are accumulated from element energies

$U_{\gamma}=\sum_{e}\left(U_{\gamma}\right)_{e}$ and $U_{\sigma}=\sum_{e}\left(U_{\sigma}\right)_{e}$
The stiffness matrices depend explicitly only on the local element density $\rho_{e}$ and if this dependence is assumed to be linear proportionality we find

$$
\begin{aligned}
\frac{\partial \lambda}{\partial \rho_{e}}= & -\frac{1}{U_{\sigma}}\left(\frac{\partial\left(\widehat{U}_{\gamma}\right)_{e}}{\partial \rho_{e}}+\lambda \frac{\partial\left(\widehat{U}_{\sigma}\right)_{e}}{\partial \rho_{e}}\right)= \\
& -\frac{1}{U_{\sigma} \rho_{e}}\left(\left(U_{\gamma}\right)_{e}+\lambda\left(U_{\sigma}\right)_{e}\right)=\frac{\left(U_{\sigma}\right)_{e}}{U_{\sigma}} \frac{1}{\rho_{e}}\left(\lambda_{e}-\lambda\right)
\end{aligned}
$$

For other dependence than linear proportionality, see Pedersen and Pedersen (2015) and Section 4.1.

\subsection{Influence from modified stiffness interpolation}

The assumed linear dependence of the element stiffness matrices on the design parameter $\rho_{e}$ may be substituted by a more general function $f\left(\rho_{e}\right)$, assumed being the same function for all elements. This involves modification of the sensitivity (16) as described in Pedersen and Pedersen (2014), but is here more simple because no kinetic energy is involved, and just a factor $\Gamma\left(\rho_{e}\right)$ is added to (16)

$\frac{\partial \lambda}{\partial \rho_{e}}=\frac{\left(U_{\sigma}\right)_{e}}{U_{\sigma}} \frac{1}{\rho_{e}} \Gamma\left(\rho_{e}\right)\left(\lambda_{e}-\lambda\right)$

where $\Gamma\left(\rho_{e}\right)$ is

$\Gamma\left(\rho_{e}\right)=\frac{\rho_{e} f^{\prime}\left(\rho_{e}\right)}{f\left(\rho_{e}\right)}$ with $f^{\prime}\left(\rho_{e}\right)=\frac{d f\left(\rho_{e}\right)}{d \rho_{e}}$

The simple result (17) is also valid for other finite element models. The applied function, named NLPI (Non Linear Penalization or Interpolation), is analytical presented in Pedersen and Pedersen (2012) with discussions relative to SIMP (Solid Isotropic Material Penalization) $f(\rho)=\rho_{e}^{\kappa_{1}}$, and also relative to another one parameter interpolation function named RAMP (Rational Approximation of Material Properties) $f(\rho)=$ $\frac{\rho}{1+\left(\kappa_{1}-1\right)(1-\rho)}$. The parameter $\kappa_{1}$ is the slope of $f$ at $\rho_{e}=1$ and for NLPI the second parameter $\kappa_{0}$ is the slope of $f$ at $\rho_{e}=0$. Figure 1 shows the interpolation function and corresponding $\Gamma$ functions for two applied cases of $\kappa_{0}, \kappa_{1}$, together with the third order SIMP penalization $f(\rho)=\rho_{e}^{3}$.

\subsection{Summary on the sensitivities}

The gradients (16) and (17) constitute the basis for an optimization procedure. They are the most valuable result of the present research and therefore presented in summary: 

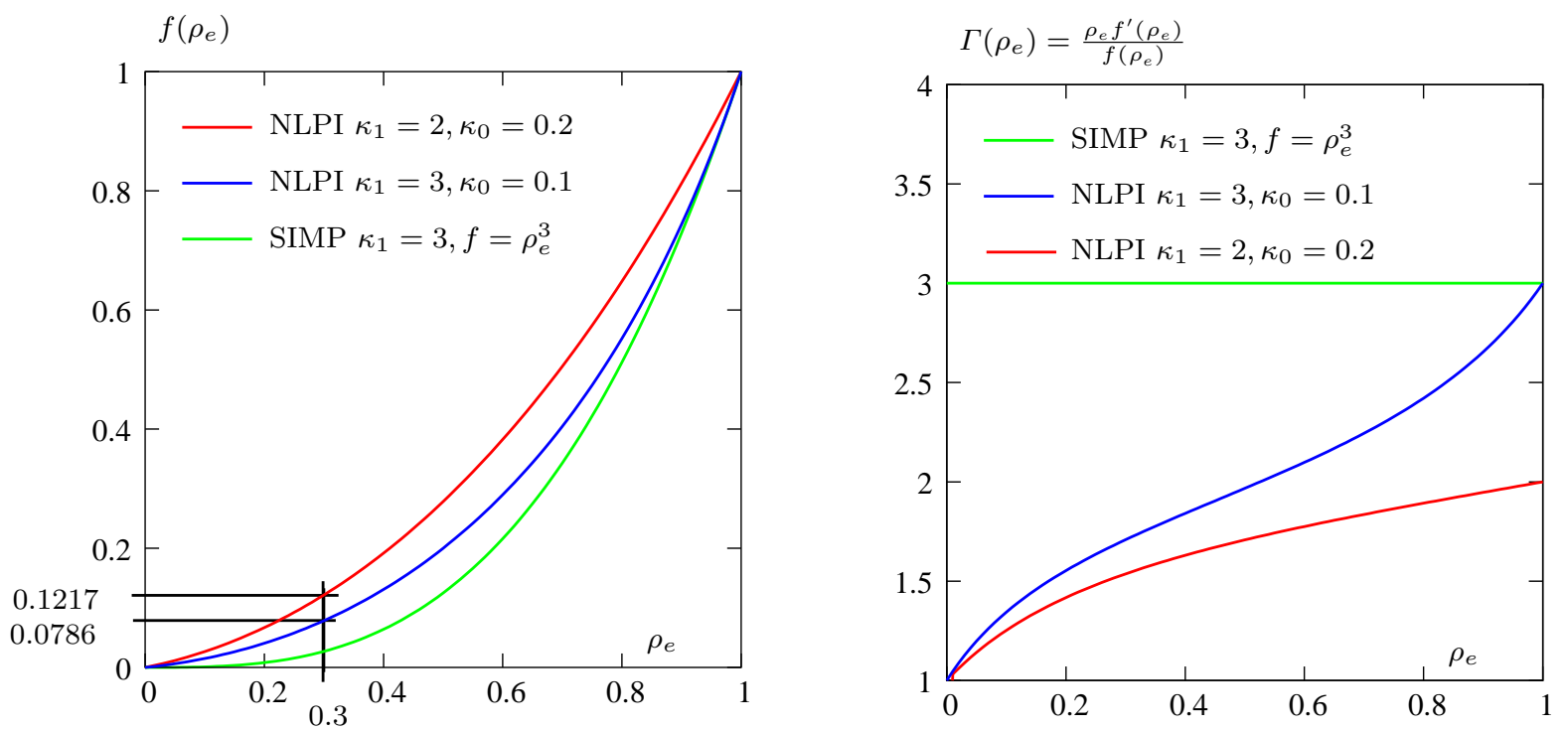

Fig. 1 Left three interpolation functions and right the corresponding factors for the sensitivity, defined in (18). The parameters $\kappa_{0}, \kappa_{1}$ are the slopes of $f$ at $\rho_{e}=0,1$. The specific values 0.1217 and 0.0786 are used in an example.

- The derivative of the critical load factor $\lambda$ with respect to density $\rho_{e}$ is determined directly by corresponding local quantities from already performed analysis.

- The derivative is proportional to the local relative energy from the stress matrix $\frac{\left(U_{\sigma}\right)_{e}}{U_{\sigma}}$.

- The derivative is inversely proportional to the local density $\rho_{e}$, for non-linear interpolation modified with the factor $\frac{\rho_{e} f^{\prime}\left(\rho_{e}\right)}{f\left(\rho_{e}\right)}$.

- The derivative is proportional to the difference between local Rayleigh quotient and system Rayleigh quotient, i.e., proportional to $\left(\lambda_{e}-\lambda\right)$.

- The sign of the derivative is equal to the sign of $\frac{\left(U_{\sigma}\right)_{e}}{U_{\sigma}}\left(\lambda_{e}-\lambda\right)$ where $U_{\sigma}$ may be normalized to 1 .

- When $\Gamma\left(\rho_{e}\right)$ is constant as in the SIMP penalization, it is just the same scaling factor on all sensitivities (and on values for optimality criterion). For the applied NLPI interpolation it influences the result of optimization.

\section{Design optimality criterion and numerical procedure}

The objective of maximizing the buckling load $\lambda_{1}\{A\}_{i}$, i.e., maximizing $\lambda_{1}$, subject to a constraint of unchanged total mass/volume is

Maximize $\lambda_{1}$ for $g=\sum_{e} \rho_{e} V_{e}-V=0$

and the necessary optimization criterion is $\frac{\partial \lambda}{\partial \rho_{e}}=\Lambda \frac{\partial g}{\partial \rho_{e}}=\Lambda V_{e} \Rightarrow$

$\frac{\left(U_{\sigma}\right)_{e}}{U_{\sigma}} \frac{\Gamma\left(\rho_{e}\right)}{\rho_{e} V_{e}}\left(\lambda_{e}-\lambda\right)=\Lambda_{e}=\Lambda$

with a constant $\Lambda$. By normalizing the buckling mode $\{\Delta\}, U_{\sigma}$ may be normalized to 1 .

\subsection{Numerical design procedure for density variables}

Assume that the value of the optimality criterion for element $e$ is termed $\Lambda_{e}$. Then in cases with negative as well as positive ratios $0>\left(\Lambda_{e}\right)_{\min } \leq \Lambda_{e} \leq\left(\Lambda_{e}\right)_{\max }>$ 0 , which is the case for the buckling optimization, the following heuristic procedure has been applied

For positive gradients $\left(\frac{\left(U_{\sigma}\right)_{e}}{U_{\sigma}}\left(\lambda_{e}-\lambda\right)>0\right)$

$$
\left(\rho_{e}\right)_{\text {new }}=\left(\rho_{e}\right)_{\text {current }}\left(1+f_{p} \frac{\Lambda_{e}}{\Lambda_{\max }}\right)^{q} \mathrm{~F}
$$

For negative gradients $\left(\frac{\left(U_{\sigma}\right)_{e}}{U_{\sigma}}\left(\lambda_{e}-\lambda\right)<0\right)$

$$
\left(\rho_{e}\right)_{n e w}=\left(\rho_{e}\right)_{\text {current }}\left(1-f_{n} \frac{\Lambda_{e}}{\Lambda_{\min }}\right)^{q} F
$$

i.e., always increase for a positive gradient and decrease for a negative gradient. The values of $\left.\Lambda_{\min }<0, \Lambda_{\max }\right\rangle$ 0 are determined during the evaluation of the gradients. Specific values in (21) $\left(f_{p}, f_{n}, q=4,0.8,0.25\right.$ or $\left.f_{p}, f_{n}, q=1,0.5,0.5\right)$ are chosen after experience with 
a given problem, acting as kind of move-limits and influence the number of recursive redesigns (number of eigenvalue analysis) with $F$ in an inner iteration determined such that the total volume constraint is exactly satisfied, see Pedersen and Pedersen (2012) for details.

For the procedure (21) the size limits of the nondimensional density variables

$0<\rho_{\min } \leq \rho_{e} \leq \rho_{\max } \leq 1$

are satisfied at each iteration in the "inner" iteration loop without further analysis and sensitivity analysis. The converged factor $F$ thereby satisfies both the size limits (22) and the specified total amount of material/volume $V$ by $\sum_{e} \rho_{e} V_{e}=V$.

The described iterative redesign procedure to increase the load level for buckling initiation is as follows:

- For a given distribution of densities, i.e., a given non-uniform $\mathrm{FE}$ model solve the non-linear analysis problem with load $\{A\}_{i}$.

- With resulting displacement gradients and resulting stresses the system matrices $\left[S_{\gamma}\right],\left[S_{\sigma}\right]$ are evaluated.

- The eigenvalue problem (8) is solved by subspace iterations to obtain the first two lowest eigenvalues and corresponding buckling eigenmodes $\lambda_{1}, \Delta_{1}$ and $\lambda_{2}, \Delta_{2}$. The design history is shown by $\lambda_{1}, \lambda_{2}$ for redesigns $0,1,2, \ldots$

- Assuming the initial load vector to be $\{A\}_{i}$, then the critical buckling load vector is $\lambda_{1}\{A\}_{i}$.

- For each element evaluate the local Rayleigh quotient $\lambda_{e}$.

- Redesign according to distribution of the optimality criterion (20) with the applied heuristic procedure (21), using a not too large value of relaxation exponent $q$, say $q=0.5$ or even $q=0.25$, for a detailed model.

\section{Numerical example}

The influence of numerical parameters for the chosen numerical approach and of the basic parameters for the design problem, i.e., the total amount of available material and of stiffness interpolation as a function of relative density can be studied in numerical examples. The example below documents the effectiveness of the approach.

\subsection{Example of a single column}

At first a column with design space of height $20 \mathrm{~m}$ and a squared cross-section of $3 \mathrm{~m} \times 3 \mathrm{~m}$ is analyzed and the buckling optimized. Figure 2 shows the discretization of the cross section and an indication for a central part that at the free top end is loaded with a uniform distributed load towards the corresponding part at the bottom end that is fixed in all three directions $x, y, z$. The remaining part of the bottom end is only fixed in the $z$-direction. The total load is $5.625 \cdot 10^{7} \mathrm{~N}$.

In the length direction the $\mathrm{FE}$ discretization is in 65 levels with each $16 \times 16$ quadratic domains, i.e., between two levels 256 box domains of each 6 tetrahedrons, as shown in Figure 3, i.e., totally 98304 tetrahedron elements. This FE model is not completely symmetric due to the division of the boxes into 6 tetrahedrons, and this means that a further imperfection is not necessary for buckling. For easier interpretation of the resulting designs and their corresponding responses, it is chosen to present smooth results where all tetrahedrons in a box after each redesign are set to the mean value of these six tetrahedrons.

Chosen parameters for the first case is:

- Stiffness interpolation by $\kappa_{0}, \kappa_{1}=1,1$, i.e., linear interpolation.

- Total amount of material by $\rho_{\text {mean }}=0.3$, i.e., $30 \%$ of maximum.

- Size constraints: $\rho_{\min }=0.1$ and $\rho_{\max }=1$

- Redesign relaxation by $q=0.5$ and $f_{p}, f_{n}=1,0.5$ in (21), giving rather stable convergence in 10-20 redesigns.

Figure 4 shows the history of buckling load factor $\lambda_{1}$ as a function of redesign number with 0 for the initial uniform design of $30 \%$ densities in the specified design space. The $\lambda_{1}$ factor for uniform design corresponds to 15.5 and by 20 redesigns this increases to 20.9 , i.e., with initial load $5.625 \cdot 10^{7} \mathrm{~N}$ the total distributed load before buckling is increased from $8.7 \cdot 10^{8} \mathrm{~N}$ to $11.7 \cdot 10^{8} \mathrm{~N}$. The influence of mode switching is clearly seen by the jagged curves.

The two lower curves of Figure 4 illustrate the total accuracy of the determined sensitivities. The obtained $\left(\Delta \lambda_{1}\right)_{o}$ is based on a smoothed model where all tetrahedrons in a box after each redesign are set to the mean value of these six tetrahedrons, and therefore with less gain. The expected $\left(\Delta \lambda_{1}\right)_{e}$ is based on individual redesign of the six tetrahedron in a box and $\left(\Delta \lambda_{1}\right)_{e}>$ $\left(\Delta \lambda_{1}\right)_{o}$ is found in most shown cases. For the final redesigns with no mode switching $\left(\Delta \lambda_{1}\right)_{e} \simeq\left(\Delta \lambda_{1}\right)_{o}$ is found.

The resulting design after 20 redesigns is illustrated in Figures 5 by density distributions at 16 levels (at every fourth level as mean values of connecting elements). A rather general interpretation is: 1) low density at the free loaded end to distribute the midpoint load towards 
loaded free end

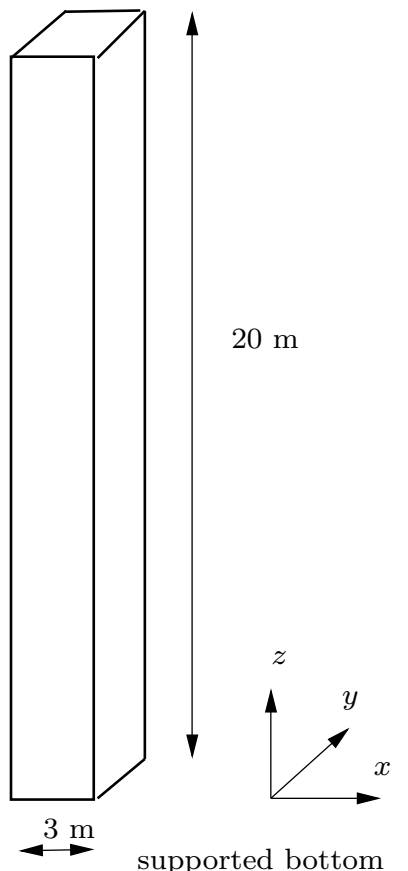

$3 \mathrm{~m}$

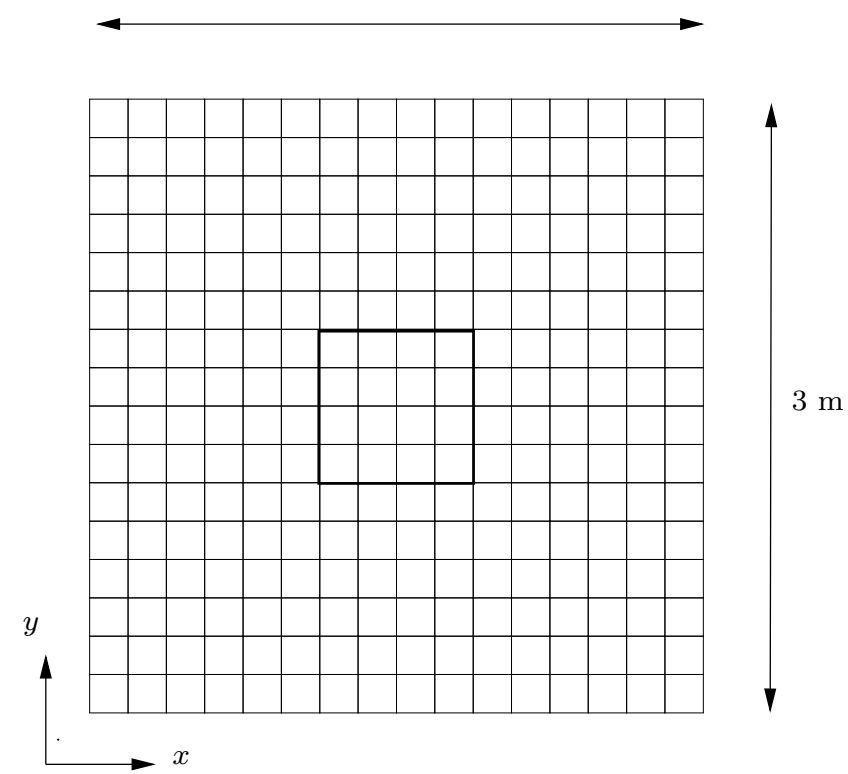

Fig. 2 Sketch of a 3D cantilever column with the finite element box discretization of the cross section. The thick lines at mid indicate the loaded domain at the free end as well as the completely fixed domain at the bottom.
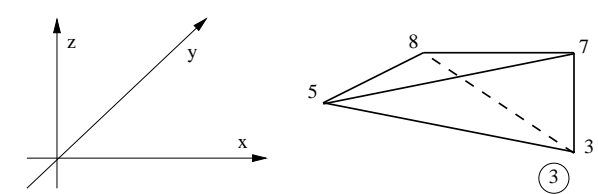

(3)
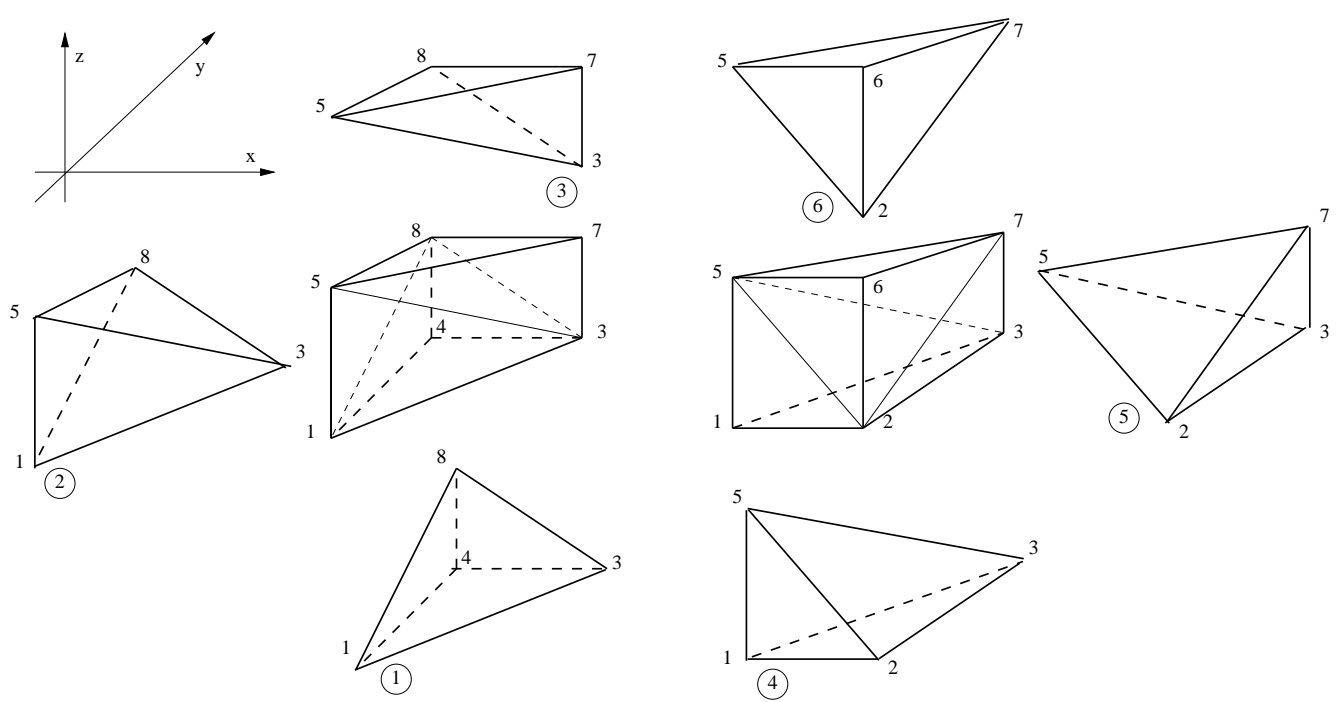

Fig. 3 Eight node hexahedron element divided first into two wedges elements and then into six tetrahedra elements, numbered in circles. The numbering of the eight nodes of the hexahedron is also related to the corner nodes of the tetrahedra.

the quadratic boundary, 2) for next quarter part higher densities at the corner gradually increasing, 3) for the third and fourth part, still low densities in the mid part also close to the fixed supports, and maximum density at the corners.

All the cross sections in Figure 5 show close to double symmetry for the material distribution. It is then expected to determine close to double eigenvalues. Figure 6 illustrate the obtained lowest buckling mode. With reference to a diagonal of the squared domains for the full continuum, the buckling mode corresponds to skew bending without torsion. The second buckling mode has reference to the other diagonal and the two eigenvalues are close as seen in Figure 4.

Figure 7 shows the distribution of optimality criterion values after 20 redesigns. Almost equal values are obtained with values close to zero, indicating that the optimality criterion is fulfilled. 


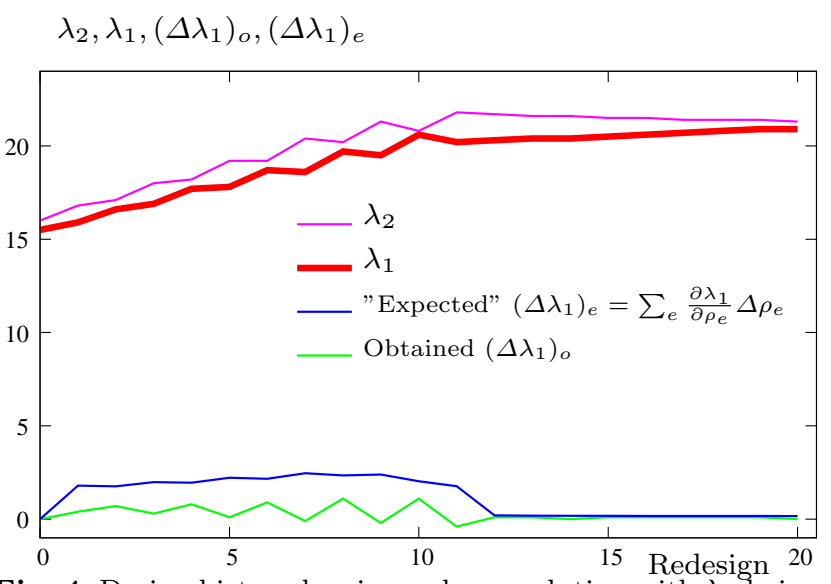

Fig. 4 Design history by eigenvalues evolution with $\lambda_{1}$ being the objective. The thick red line correspond to $\lambda_{1}$ while the lighter line correspond to $\lambda_{2}$. Interpolation by $\kappa_{0}, \kappa_{1}=1,1$, i.e., linear interpolation. Total material $30 \%$. Obtained increments by green line and expected increments by blue line. The "shared" point for $\lambda_{1}$ and $\lambda_{2}$ indicate close eigenvalues, but only one buckling mode is the basis for the following redesign.
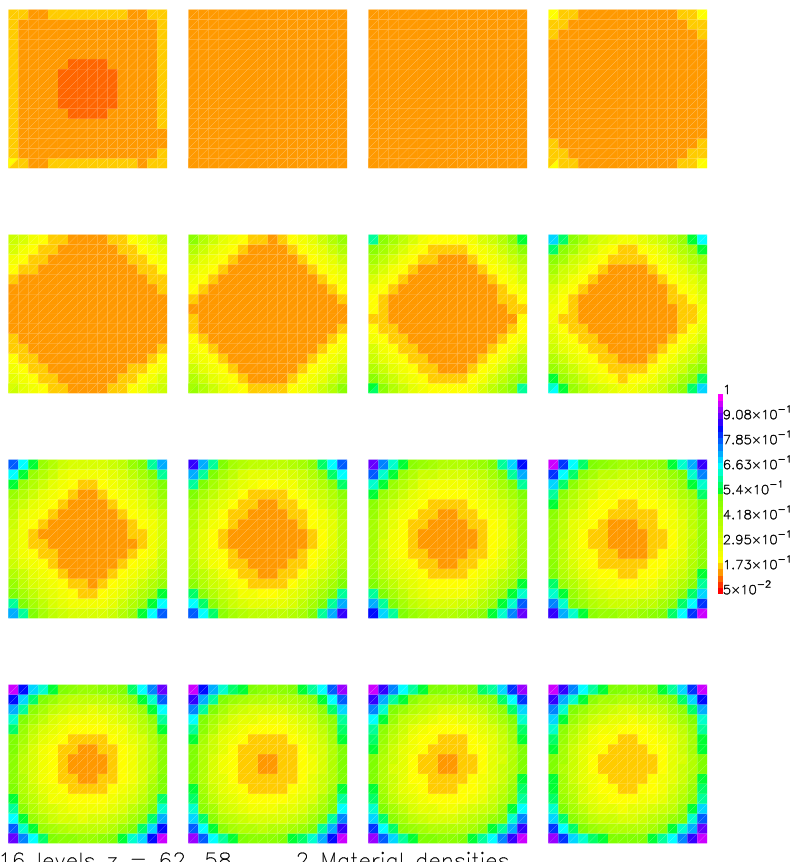

Fig. 5 Design after 20 redesigns with interpolation by $\kappa_{0}, \kappa_{1}=1,1$, i.e., stiffness proportional dependent on the local density parameter $\rho_{e}$. Starting from upper left corner close to the free, loaded end. Ending at the lower right corner close to the support.

The second case corresponds to non-linear interpolation with $\kappa_{0}, \kappa_{1}=0.2,2$ and Figure 8 shows the history of buckling load factor $\lambda_{1}$ as a function of redesign number with 0 for the initial uniform design of $30 \%$ in the specified design space. The $\lambda_{1}$ factor for
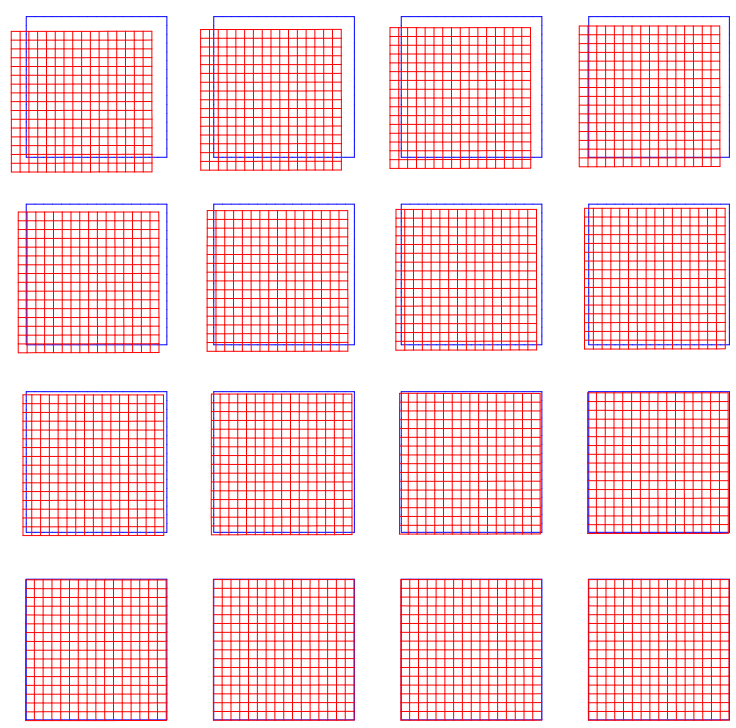

Lowest buckling mode

$\mathrm{x}, \mathrm{y}$ displacements at $16 \mathrm{z}$ levels

Fig. 6 The buckling mode displacements at every fourth $\mathrm{z}$ level. Starting from upper left corner close to the free, loaded end. Ending lower right corner close to the support. In 3D showing a skew bending without torsion. The second mode similar, but relative to the other diagonal.
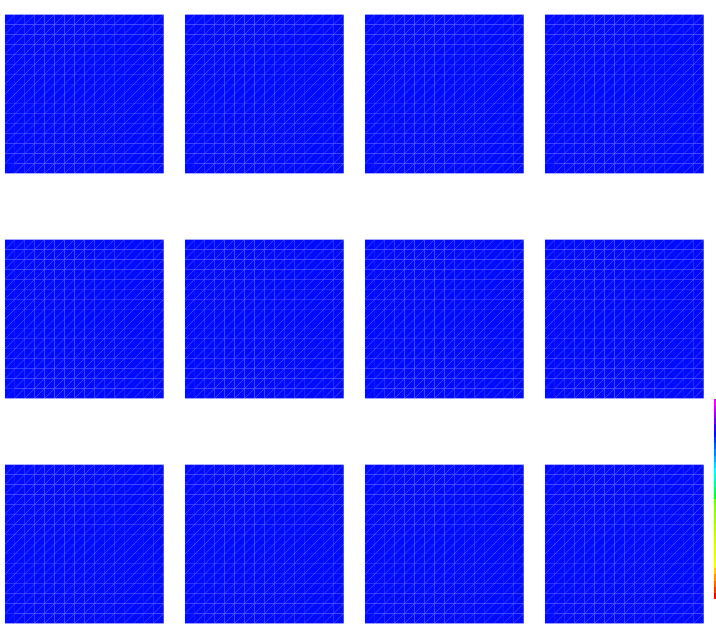

$4.98 \times 10^{1} \quad \mathrm{~m}^{-3}$ $1.28 \times 10^{1}$ $-3.65 \times 10^{1}$ $-8.58 \times 10^{1}$ $-1.351 \times 10^{2}$ $-1.844 \times 10^{2}$ $-2.337 \times 10^{2}$ $-2.83 \times 10^{2}$
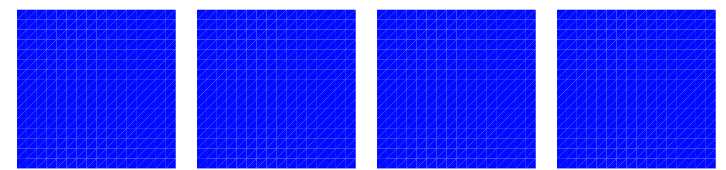

16 levels $z=62,58, \ldots ., 2$ Optimality criterion values

Fig. 7 Distribution of optimality criterion values after 20 redesigns with interpolation by $\kappa_{0}, \kappa_{1}=1,1$, i.e., stiffness proportional dependent on the local density parameter $\rho_{e}$. The figure illustrates that the optimality criterion is fulfilled. 
uniform design corresponds to 6.28 and by 20 redesigns this increases to 8.62 .

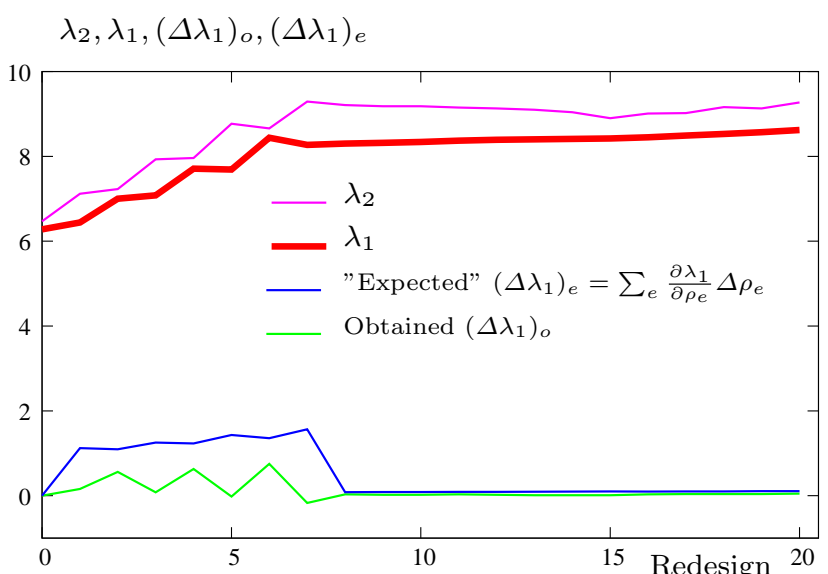

Fig. 8 Design history by eigenvalues evolution with $\lambda_{1}$ being the objective. The thick red line correspond to $\lambda_{1}$ while the lighter line correspond to $\lambda_{2}$. Interpolation by $\kappa_{0}, \kappa_{1}=0.2,2$. Total material $30 \%$. Obtained increments by green line and expected increments by blue line.

For this case of non-linear interpolation with $\kappa_{0}, \kappa_{1}=$ $0.2,2$ the resulting design after 20 redesigns is illustrated in Figures 9 by the distribution of density distributions at 16 levels. The resulting distribution of optimality criterion values is similar to Figure 7 and therefore not shown. This also holds for the following third case.
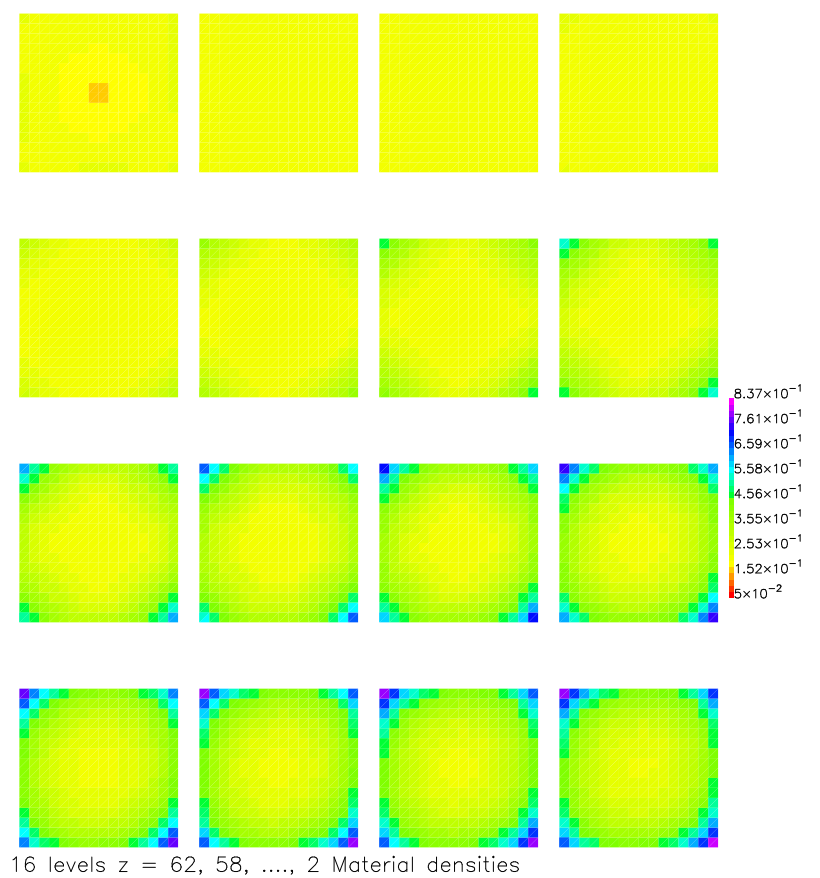

Fig. 9 Design after 20 redesigns with interpolation by $\kappa_{0}, \kappa_{1}=0.2,2$.
The third case corresponds to a stronger nonlinear interpolation with $\kappa_{0}, \kappa_{1}=0.1,3$ and Figure 10 shows the history of buckling load factor $\lambda_{1}$ as a function of redesign number with 0 for the initial uniform design of $30 \%$ in the specified design space. The $\lambda_{1}$ factor for uniform design corresponds to 4.06 and by 20 redesigns this increases to 5.61 .

$$
\lambda_{2}, \lambda_{1},\left(\Delta \lambda_{1}\right)_{o},\left(\Delta \lambda_{1}\right)_{e}
$$

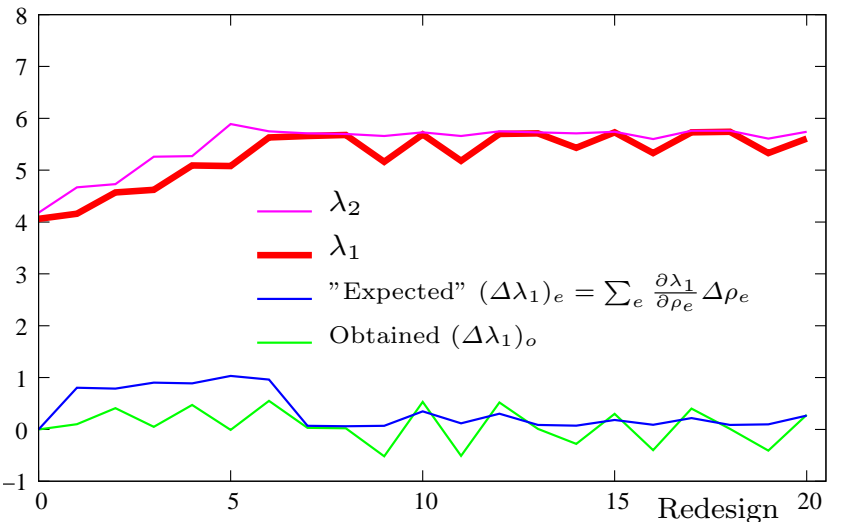

Fig. 10 Design history by eigenvalues evolution with $\lambda_{1}$ being the objective. The thick red line correspond to $\lambda_{1}$ while the lighter line correspond to $\lambda_{2}$. Interpolation by $\kappa_{0}, \kappa_{1}=0.1,3$. Total material $30 \%$. Obtained increments by green line and expected increments by blue line.

For this case of stronger non-linear interpolation with $\kappa_{0}, \kappa_{1}=0.1,3$ the resulting design after 20 redesigns is illustrated in Figures 11 by the distribution of density distributions at 16 levels.

All three Figures 4, 8 and 10 show clear convergence in spite of mode switching for first and second eigenvalue. For linear interpolation, initial to optimized values are $\lambda_{1}=15.5 \Rightarrow 20.1$; for $\kappa_{0}, \kappa_{1}=0.2,2$ interpolation, initial to optimized $\lambda_{1}=6.28 \Rightarrow 8.62$ and for $\kappa_{0}, \kappa_{1}=0.1,3$ interpolation, initial to optimized $\lambda_{1}=4.06 \Rightarrow 5.61$.

Figure 12 combines results for history of the eigenvalues. With stronger non-linear interpolation several of the lowest eigenvalues are close and the numerical analysis is more sensitive, but still solvable.

For the three cases the resulting designs after 20 redesigns are illustrated in Figures 5, 9 and 11 by the distribution of density distributions at 16 levels (at every fourth level as mean values of connecting elements). The interpretation of these figures is similar to the comments given to Figure 5. With stronger non-linear interpolation the resulting designs shows clearer concentrations of material and larger flexible domains.

For uniform density, i.e., redesign 0 in Figure 12 we may see interpolation as a scaling of stiffnesses and thereby get an estimate of the change in load factor 

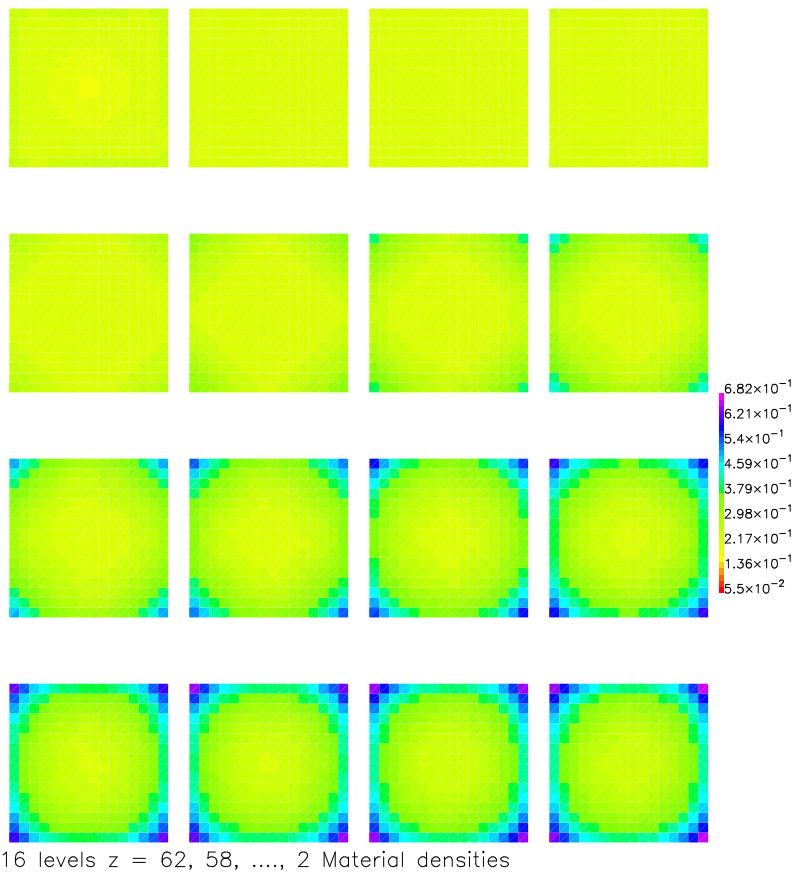

Fig. 11 Design after 20 redesigns with interpolation by $\kappa_{0}, \kappa_{1}=0.1,3$

$\lambda_{1}$ and $\lambda_{2}$

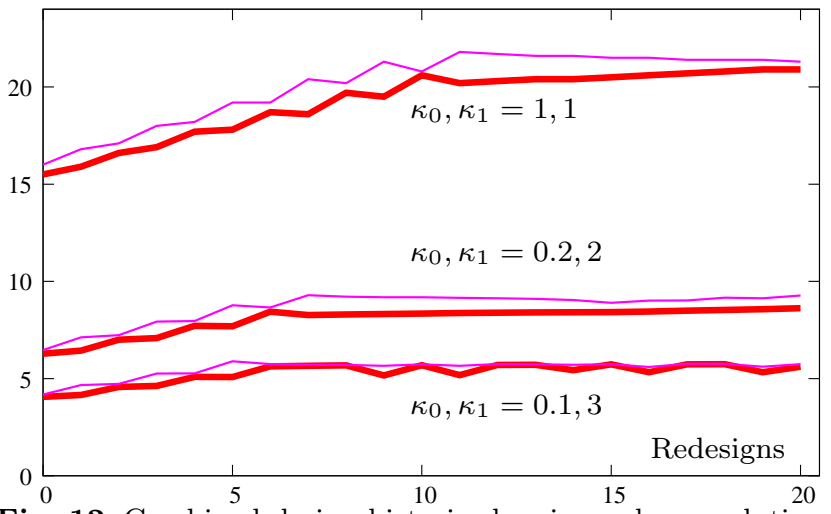

Fig. 12 Combined design histories by eigenvalues evolution with $\lambda_{1}$ being the objective. The thick lines correspond to $\lambda_{1}$ while the lighter lines correspond to $\lambda_{2}$. Three different interpolations and total material $30 \%$ for all three cases.

$\lambda_{1}$. From Figure 1 the $\lambda_{1}$ factor for $\kappa_{0}, \kappa_{1}=0.2,2$ is reduced by a factor $0.1217 / 0.3=0.406$, that estimate $\lambda_{1}$ to $15.5 \cdot 0.406=6.293$, close to the determined factor 6.28. Similar for $\kappa_{0}, \kappa_{1}=0.1,3$ with value from Figure 1 the $\lambda_{1}$ factor is $15.5(0.0786 / 0.3)=4.061$ that agree with the determined factor 4.06 . The strong influence of the interpolation is illustrated in the combined Figure 12 , not only by the scaling, which itself is depending on the total amount of material, but also by the closeness of eigenvalues and the mode switching.

\section{Conclusions}

For a four node tetrahedron element, a simple evaluation of the stress stiffness matrix is presented and applied in buckling analysis that is formulated as an eigenvalue problem. Based on static non-linear equilibrium the eigenvalue problem is set up and solved by subspace iterations.

The simple sensitivity analysis is obtained on the basis of a linear extrapolation from a geometrical nonlinear static equilibrium, and give rice to rather robust optimizations. Localized sensitivities are analytically obtained and this makes optimization possible also for large (100000) numbers of local design variables. Cases of optimization for a single non cylindrical column are presented. The influence of chosen stiffness interpolations is shown.

Although the multi-mode aspects are important, it is here decided to investigate the limitations for redesign based only on the mode associated with the lowest eigenvalue. Also in relation to mode switching this redesign approach is found practical and without divergent solutions.

\section{References}

Bruyneel M, Colson B, Remouchamps A (2008) Discussion on some convergence problems in buckling optimisation. Struct Multidisc Optim 35(2):181-182

Colson B, Bruyneel M, Grihon S, Raick C, Remouchamps A (2010) Optimization methods for advanced design of aircraft panels: a comparison. Optim Eng 11:583-596

Cook RD, Malkus DS, Plesha ME, Witt RJ (2002) Concepts and Applications of Finite Element Analysis, 4th edn. Wiley, New York, USA, 719 pages

Crisfield MA (1991 and 1997) Non-linear Finite Element Analysis of Solids and Structures, vol 1 and 2. Wiley, Chichester, UK, 345 and 494 pages

Dunning PD, Ovtchinnikov E, Scott J, Kim HA (2016) Level-set topology optimization with many linear buckling constraints using an efficient and robust eigensolver. Int J Num Meth Eng 107(12):1029-1053

Haftka RT, Gurdal Z, Kamat MP (1990) Elements of Structural Optimization. Kluwer, Dordrecht, The Netherlands, 396 pages

Kleiber M, Hien TD (1997) Parameter sensitivity of inelastic buckling and post-buckling response. Comput Methods Appl Mech Engrg 145:239-262

Luo Q, Tong L (2015) Structural topology optimization for maximum linear buckling loads by using a moving iso-surface threshold method. Struct Multidisc Optim 52:71-90 
Mróz Z, Haftka RT (1994) Design sensitivity analysis of non-linear structures in regular and critical states. Int JSolids Structures 31(15):2071-2098

Ohsaki M (2005) Design sensitivity analysis and optimization for nonlinear buckling of finite-dimensional elastic concervative structures. Comput Methods Appl Mech Engrg 194:3331-3358

Pedersen P (2006) Analytical stiffness matrices for tetrahedral elements. Computer Methods in Applied Mechanics and Engineering 196:261-278

Pedersen P, Pedersen NL (2012) Interpolation/penalization applied for strength designs of $3 \mathrm{~d}$ thermoelastic structures. Struct Multidisc Optim 45:773-786

Pedersen P, Pedersen NL (2014) A note on eigenfrequency sensitivities and structural eigenfrequency optimization based on local sub-domain frequencies. Struct Multidisc Optim 49(4):559-568

Pedersen P, Pedersen NL (2015) Eigenfrequency optimized 3D continua, with possibility for cavities. Journal of Sound and Vibration 341:100-115

Sørensen SN, Sørensen R, Lund E (2014) DMTO - a method for discrete material and thickness optimization of laminated composite structures. Struct Multidisc Optim 50:25-47

Wittrick WH (1962) Rates of change of eigenvalues, with reference to buckling and vibration problems. J Royal Aeronautical Soc 66:590-591

Wu CC, Arora JS (1988) Design sensitivity analysis of non-linear buckling load. Comput Mech 3:129-140 